\title{
APLICAÇÃO DE INSTRUMENTO HÍBRIDO DE ANÁLISE DE MODOS DE FALHA E EFEITOS NA DOCAGEM DE EMBARCAÇÕES EM DIQUES SECOS
}

\author{
Milton George Fonseca Kämpffe \\ Universidade Federal Fluminense (UFF) \\ Programa de Pós-Graduação em Engenharia de Produção \\ Rua Passo da Pátria, 156, São Domingos, Niterói, RJ \\ milton_kampffe@id.uff.br \\ Gilson Brito Alves Lima \\ Universidade Federal Fluminense (UFF) \\ Programa de Pós-Graduação em Engenharia de Produção \\ Rua Passo da Pátria, 156, São Domingos, Niterói, RJ \\ glima@id.uff.br
}

Annibal Parracho Sant'Anna

Universidade Federal Fluminense (UFF)

Programa de Pós-Graduação em Engenharia de Produção

Rua Passo da Pátria, 156, São Domingos, Niterói, RJ

annibal.parracho@gmail.com

Isaac José Antonio Luquetti dos Santos

Instituto de Engenharia Nuclear (IEN)

Comissão Nacional de Engenharia Nuclear (CNEN)

Rua Hélio de Almeida, 75, Ilha do Fundão, Rio de Janeiro, RJ isaacluquetti@gmail.com

\section{Luiz Carlos Delgado}

Centro Tecnológico da Marinha no Rio de Janeiro (CTMRJ) Rua Ipiru, 2, Cacuia, Ilha do Governador, Rio de Janeiro, RJ ctmrj-01@marinha.mil.br

\section{Leonardo George da Silva Kämpffe}

Escola Naval (EN)

Av. Almirante Silvio de Noronha, s/n, Castelo, Rio de Janeiro, RJ kampffe97@gmail.com 


\title{
RESUMO
}

A docagem é um evento de suma importância para a vida operacional de um navio e ocorre por força de cumprimento das regras internacionais de segurança e de meio ambiente emanadas pela International Maritime Organization (IMO). Neste sentido, o objetivo deste artigo é revisitar, decorridos cerca de quatro anos, por meio de entrevistas realizadas aos especialistas responsáveis pela área de docagem, os riscos atualmente existentes em operações de docagem de navios, especificamente no mesmo dique seco estudado no ano de 2015. A abordagem metodológica consistiu na aplicação das técnicas de identificação de perigos e FMEA na obtenção de números de prioridade de risco (RPN), e na posterior classificação dos riscos identificados, com uma melhor priorização entre eles para tratamento, através do emprego do método da Composição Probabilística de Preferência (CPP). Como resultado decorrente da pesquisa realizada, observou-se que a aplicação deste método gerou valores que propiciaram uma melhor priorização para tratamento dos riscos considerados inaceitáveis, quando comparado ao calculo do RPN da FMEA clássica e uma robustez ao processo de tomada de decisões nos cenários de docagem de navios.

Palavra-chave: Docagem; Risco; Confiabilidade; FMEA; CPP; Dique Seco.

\begin{abstract}
Dockage is an event of paramount importance for the operational life of a ship and is carried out in compliance with the International Maritime Organization's (IMO) international safety and environmental standards. In this sense, the objective of this article is to revisit, after approximately four years, through interviews with specialists responsible for the docking area, the risks currently present in ship docking operations, specifically in the same dry dock studied in the year 2015. The methodological approach consisted in the application of the techniques of hazard identification and Analysis of Failure Modes and Effects (FMEA), in obtaining Risk Priority Numbers (RPN), and in the subsequent classification of identified risks, with a better prioritization among them for treatment, through the use of the Probabilistic Composition of Preferences (CPP) method. As a result of the research, it was observed that the application of CPP generated values that provided a better prioritization for the treatment of the risks considered unacceptable, when compared to the RPN of the FMEA and a robust decision-making process in ship docking scenarios.
\end{abstract}

Keywords: Docking; Risk; Reliability; FMEA; CPP; Dry Dock.

\section{Como Citar:}

KÄMPFFE, Milton George Fonseca et al. Aplicação de instrumento híbrido de análise de modos de falha e efeitos na docagem de embarcações em diques secos. In: SIMPÓSIO DE PESQUISA OPERACIONAL E LOGÍSTICA DA MARINHA, 19, 2019, Rio de Janeiro, RJ. Anais do XIX Simpósio de Pesquisa Operacional e Logística da Marinha. Rio de Janeiro: Centro de Análises de Sistemas Navais, 2019. 


\section{INTRODUÇÃO}

Os portos são a principal porta do Brasil para o mundo, por onde passam 95\% do comércio exterior brasileiro [2].

O mar é a nossa principal via de transporte. A indústria nacional importa matériaprima e exporta seus produtos essencialmente através do transporte marítimo.

A área de logística, um dos elos da cadeia produtiva do transporte marítimo, possui projetos caracterizados pela manutenção preventiva dos navios existentes, que emprega a docagem, e pela construção de novos navios [13].

Dentre os eventos relativos à conservação, a docagem é de suma importância para a vida operacional de um navio e, no caso da frota mercante, ocorre por força de cumprimento das regras internacionais de segurança e de meio ambiente emanadas pela International Maritime Organization (IMO).

A originalidade da proposta desenvolvida inicialmente por [8] se contextualizou em um instrumento hibrido FMEA-CPP como ferramenta de apoio à decisão, na obtenção da priorização para o tratamento dos riscos considerados inaceitáveis relativos à docagem de embarcações em diques secos.

A proposta deste artigo é revisitar, decorridos cerca de quatro anos, por meio de entrevistas realizadas aos especialistas responsáveis pela área de docagem, os riscos atualmente existentes em operações de docagem de navios, especificamente no mesmo dique seco estudado no ano de 2015. Foram empregadas por [8] as técnicas de identificação de perigos e Análise de Riscos Qualitativa de Modos de Falha e Efeitos (FMEA) e da Composição Probabilística de Preferência (CPP).

\section{METODOLOGIA DE PESQUISA}

A estrutura do método de pesquisa está representada em cinco passos na Figura 1 e nos seus desdobramentos expostos a seguir.

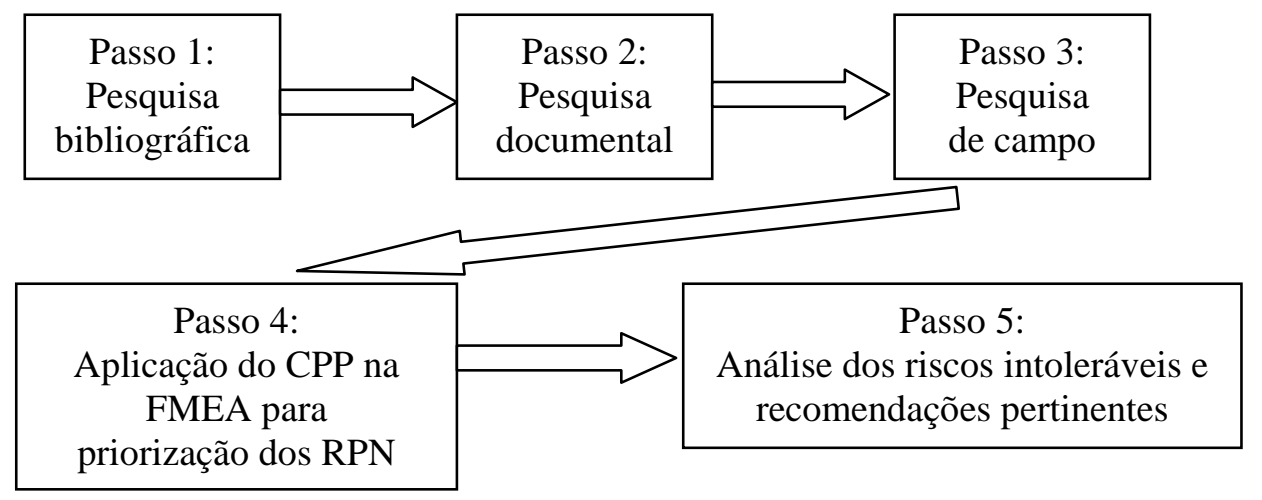

Figura 1 - Processo metodológico aplicado Fonte: [8]

O caminho metodológico utilizado neste artigo é descrito a seguir. Como passo inicial, a fim de obtermos resultados que conduzissem a uma massa critica de trabalhos científicos sobre o tema de interesse realizou-se uma pesquisa bibliográfica no portal de periódicos da Coordenação de Aperfeiçoamento de Pessoal de Nível Superior (CAPES).

Os critérios de seleção de trabalhos científicos foram os seguintes: (1) Foco em estudos que contivessem pelo menos as palavras chave risk management, risk analysis, risk 
assessment, docking, inspection, maintenance, planning, procedure, repair, security, ship e shipyard em seu título, resumo ou palavras chave; um segundo levantamento foi realizado com as seguintes palavras: evaluation, interview, questionary, questionnaire, research, statistical inference, statistical survey, survey, failure mode, fmea e fmeca; (2) Trabalhos disponíveis online; (3) Trabalhos escritos em Português ou Inglês; (4) Trabalhos que discutiam o tema deste artigo em outros campos diferentes de engenharia, administração de empresas ou ciências sociais foram excluídos. Um aspecto a ser destacado é que os critérios de análise estão circunscritos a efeitos de severidade de caráter não financeiro.

Em um segundo passo, foram levantados, através de literatura específica, os modos de falha, causas e efeitos de eventos de risco e componentes mecânicos utilizados nas operações de docagem de embarcações em diques secos.

No passo três foi realizada a aplicação de um instrumento de coleta de dados (survey) com especialistas em docagem e posterior validação que forneceu subsídios para a construção de planilhas de FMEA.

Segundo [6], as pesquisas deste tipo são caracterizadas pela interrogação direta das pessoas cujo comportamento se deseja conhecer. Solicita-se informações a um grupo significativo de pessoas acerca do problema estudado para em seguida, mediante análise quantitativa, obter as conclusões correspondentes dos dados coletados.

No quarto passo os riscos identificados foram hierarquizados para tratamento a partir da aplicação do método da composição probabilística de preferência (CPP).

No último passo, a partir das análises realizadas, foram descritas ações recomendadas para o tratamento dos riscos considerados inaceitáveis.

\subsection{ANÁLISE DE MODOS DE FALHA E EFEITOS}

Existem muitas definições de risco, mas a maioria delas envolve a noção de um resultado adverso ou um impacto de potencial negativo que surge de algum processo presente ou evento futuro. A ocorrência do evento é possível e não certa, seja porque é desconhecida ou porque ocorre com alguma probabilidade. Significando também que a perda é provável e não certa [7].

Risco é um conceito com diversos significados, que depende do contexto e da disciplina científica nas quais o termo é utilizado. Em seu sentido geral, risco designa a possibilidade de perda. O termo é, em geral, usado para designar conhecimento de parâmetros de uma distribuição de probabilidades, num conjunto de acontecimentos alternativos [11].

A Análise de Modos de Falha e Efeitos, Failure Modes and Effects Analysis (FMEA), avalia e classifica cada modo de falha potencial em um sistema para determinar os efeitos críticos de acordo com a sua severidade, ocorrência e detectabilidade [23]. É utilizada para determinação de problemas originados de produtos e processos, e pode ser aplicada em vários níveis, como componentes, equipamentos ou sistemas, dependendo do grau de detalhamento desejado.

Embora seja dada ênfase a identificação dos problemas resultantes de falhas dos equipamentos, as ações humanas que causem falha dos componentes também devem ser consideradas.

Em sua execução, a FMEA requer o envolvimento de um grupo de especialistas como: gerentes de qualidade, gerentes técnicos, engenheiros de manutenção e de segurança [3].

Segundo [23], a FMECA, Failure Modes and Effects Criticality Analysis distinguese da FMEA pelo fato de agregar o índice de criticidade para priorizar os riscos e orientar nas ações a serem executadas pela organização. De maneira análoga, [10] menciona que a 
FMECA seria uma otimização da FMEA, onde é realizada uma análise crítica atribuindo a cada modo de falha um valor de frequência, e a cada efeito de falha um valor de severidade. Por conveniência, neste artigo será adotado o termo FMEA indistintamente, sendo considerada, no processo de análise, a criticidade dos modos de falha e dos efeitos.

A análise crítica do sistema é realizada levando em conta os fatores de ocorrência (O), severidade (S) e detecção (D) levantados. A priorização dos modos de falha para a aplicação de ações corretivas é feita através do cálculo do Risk Priority Number (RPN), que é obtido pela multiplicação dos valores O, S e D de um modo de falha [12].

Para cada modo de falha, S, O e D são avaliados em uma escala de pontos e, quanto maior for o valor do RPN, maior será o risco para o sistema.

\subsection{COMPOSIÇÃO PROBABILÍSTICA}

As críticas mais importantes que a FMEA tem recebido decorrem de que ao combinar as avaliações do risco segundo cada critério não se leva em conta a variabilidade observada em cada critério nem a incerteza inerente a essas avaliações segundo cada critério. Assim, por exemplo, quando todas as causas são avaliadas segundo um dos critérios em níveis muito baixos e segundo o outro critério em níveis muito altos, diferenças na avaliação segundo o primeiro critério resultarão em diferenças maiores no índice final do que diferenças na avaliação segundo o outro.

Em [17] se propõe a utilização da Composição Probabilística para a determinação do risco associado a cada evento, em substituição a multiplicação direta dos índices atribuídos. Na transformação probabilística, para os valores de severidade, ocorrência e detecção são realizados os cálculos dos vetores probabilidade de preferência para distribuições de probabilidade próprias e, em seguida, o cálculo da prioridade global. Os riscos classificados com alta prioridade, pela composição probabilística, seriam então avaliados para tratamento, com base na análise de especialistas em cada área.

Com as probabilidades de maximizar a preferência segundo cada critério, para se compor uma medida única de preferência global, basta considerar essas probabilidades como condicionais na escolha dos respectivos critérios. Neste caso, a medida global será dada pela soma dos produtos das probabilidades de preferência conforme cada critério pelas probabilidades dele ser escolhido.

A dificuldade prática em validar esta medida advém apenas de comparação dos critérios. Se for possível atribuir a cada critério uma medida de preferência, podemos obter os pesos desejados através do cálculo das probabilidades de maximizar as preferências, tratando os critérios como opções quaisquer entre as quais se procede a uma escolha.

Em [18] se sugere calcular um conjunto de outras probabilidades de maximização conjunta da preferência. Cada uma dessas medidas globais decorre de um enfoque diferente quanto à combinação das probabilidades de maximizar ou minimizar as preferências segundo os vários critérios. Amplos detalhes sobre o uso da CPP encontram-se em [16, 19 e 20].

No presente artigo será empregado o CPP para a priorização no tratamento dos riscos identificados, obtendo-se uma maior confiabilidade, quando comparada à ordenação utilizando-se o RPN, no qual, em muitos casos, poderia haver empates.

\subsection{DOCAGEM}

A docagem é uma etapa fundamental para a construção, reparo e a vida operacional de um navio. A sua consecução requer cuidados especiais devido aos diversos riscos envolvidos para a segurança dos trabalhadores e do material. Existem cinco tipos de diques: 
seco (graving docks); flutuante (floating docks); Syncrolifts; Hidrolift e de carreira (slipways).

No Brasil, os mais utilizados para reparos e docagens são os diques seco e flutuantes. Em virtude da abrangência do assunto e da diversidade de tipos, a abordagem deste artigo foi delimitada aos diques secos.

Foi realizada uma busca pelo significado desta palavra em dicionários da língua portuguesa. Em [4] dique seco é definido como um tanque que pode ser esvaziado para reparo no casco de navios e doca como um dique para construção ou reparo de tais embarcações, enquanto [5] esclarece que dique é um reservatório circundado de paredes sólidas em comunicação com o mar ou rio, no qual se pode regular por uma porta a entrada e saída das águas, para nele se introduzir embarcações, pô-las em seco para reparos no casco e de novo alagá-las para saída da embarcação.

O dique seco localiza-se em terra firme, pode se encher e esvaziar de água e é destinado a receber navios cujo casco deva ser conservado ou reparado na seguinte sequencia de procedimentos: 1 - Enchimento do dique por gravidade; 2 - Abertura da comporta (porta-batel); 3 - Entrada do navio; 4 - Fechamento da comporta e esgotamento do dique por bombas; e 5 - Assentamento nos picadeiros [22].

Os procedimentos de entrada e saída do dique são constituídos, principalmente, de uma sequência de atividades que visam a garantir a segurança das manobras por meio da correta utilização dos recursos humanos e materiais.

A entrada do navio no dique deve ser feita com um agente de manobras a bordo, o navio deve estar perfeitamente alinhado à linha de centro do dique e depois ser deslocado para o seu interior, com o auxílio de uma robusta espia central e espias laterais. Estas são movimentadas por cabrestantes instalados ao longo da borda do dique, que devem periodicamente ser mantidos e eventualmente modernizados.

A desdocagem, igualmente, deve ocorrer de forma cautelosa, com condições favoráveis de calado, maré alta e vento de baixa intensidade, a fim de minimizar as forças atuantes no través. $\mathrm{O}$ navio é retirado do dique tracionado por rebocadores e auxiliado por espias, que permitem um controle melhor do seu movimento lateral. Importante observarmos a relevância da utilização de defensas laterais localizadas em pontos estrategicamente escolhidos, que atuam de forma a impedir o contato do casco com as paredes do dique.

\section{RESULTADOS E DISCUSSÃO}

Inicialmente foram propostos a 32 especialistas (população, sem seleção de amostra) 75 eventos de risco típicos de uma docagem em dique seco em diversos contextos, conforme detalhado em [9]. O começo foi realizado com os riscos de gestão da segurança e com o aditamento da categorização proposta por [15]: Riscos Técnicos, Riscos Externos, Riscos Organizacionais e Risco de Gerenciamento de Projeto [13].

Os especialistas eram os funcionários da linha de frente, líderes e gestores que trabalham e estão distribuídos entre as Divisões de Planejamento Técnico e de Execução, que fazem parte do Departamento de Docagem de um estaleiro nacional. Estes eram distribuídos entre os níveis superior, técnico e artesanal, de diversas especialidades, com diferentes tempos de experiência. Suas especializações consistiam em: mecânica (47\%), naval/produção (38\%), elétrico-eletrônica (6\%), segurança do trabalho (6\%) e controle/garantia da qualidade (3\%). Quanto ao tempo de experiência em atividades afins de docagem observou-se a seguinte distribuição: até 7 anos (63\%), de 8 a 15 (16\%), de 16 a 23 (6\%), de 24 a 30 (9\%) e mais de 30 (6\%). Nenhum dado foi descartado, pois todos os entrevistados preencheram diretamente todos os campos das 40 perguntas do questionário e 
assinalaram somente uma resposta entre as 5 alternativas apresentadas por pergunta, sem a ocorrência de resposta em branco.

Em seguida, solicitou-se que os especialistas priorizassem os eventos considerados mais críticos. O Quadro 1 esboça os oito eventos de risco (ER) destacados pelos especialistas e suas respectivas ocorrências.

Quadro 1 - Ordem dos 75 eventos de risco do processo de docagem

\begin{tabular}{|lccc|}
\hline \multicolumn{1}{c}{ EVEN TOS DE RISCO } & OCORR. & PERCENTAGEM & ACUMULADO \\
\hline Falha no sistema de bombeamento do dique & 14 & $43,75 \%$ & $\mathbf{4 3 , 7 5 \%}$ \\
\hline Materiais não serem entreques no prazo solicitado & 6 & $18,75 \%$ & $\mathbf{6 2 , 5 0 \%}$ \\
\hline Acidente por colisão do navio & 3 & $9,38 \%$ & $\mathbf{7 1 , 8 8 \%}$ \\
\hline Atraso na execuç̃̃o dos serviços & 3 & $9,38 \%$ & $\mathbf{8 1 , 2 5 \%}$ \\
\hline Não obter condições de lastro para docagem & 3 & $9,38 \%$ & $\mathbf{9 0 , 6 3 \%}$ \\
\hline Acidentes de trabalho no estaleiro & 1 & $3,13 \%$ & $\mathbf{9 3 , 7 5 \%}$ \\
\hline Dificuldade de comunicação (idioma estrangeiro) & 1 & $3,13 \%$ & $\mathbf{9 6 , 8 8 \%}$ \\
\hline Impossibilidade de entrada ou saida (maré, vento) & 1 & $3,13 \%$ & $\mathbf{1 0 0 , 0 0 \%}$ \\
\hline Eventos de risco restantes (67) & 0 & $0,00 \%$ & $\mathbf{1 0 0 , 0 0 \%}$ \\
\hline
\end{tabular}

Fonte: [8]

Dentre os diversos critérios existentes para seleção dos eventos de risco mais expressivos, destaca-se a "lei de Pareto" (LP), que afirma que em certas situações se observa uma distribuição estatística em que $20 \%$ da população reúne $80 \%$ da riqueza total [21].

A LP é hoje utilizada no controle industrial da qualidade onde se observa que a distribuição de frequência do número de defeitos de produção versus as causas que os produzem segue uma curva aproximadamente do tipo exponencial. Alguns poucos defeitos de um agrupamento são responsáveis pela maior parte das ocorrências, enquanto a maior parte contribui muito pouco para o efeito total.

O Quadro 1 ilustra a relação entre os eventos de risco, números de ocorrências e percentagem acumulada e permite uma conclusão de grande valor prático: 90,63\% das ocorrências (percentagem acumulada) são provocadas pelos cinco primeiros eventos de risco. Neste sentido, a LP fornece uma alternativa estratégica para a escolha dos riscos e o plano de ação para o tratamento destes é facilitado: em vez de se atuar sobre todos os eventos, procura-se agir sobre os mais importantes. Objetiva-se obter assim, o máximo de resultado com o mínimo gasto de recursos.

Como critério de decisão, foram selecionados, como os mais significativos para serem estudados mais detalhadamente, os cinco primeiros eventos com riscos mais elevados, com os seguintes códigos: ER1 - Falha no sistema de bombeamento do dique, ER2 Materiais não serem entregues no prazo solicitado, ER3 - Acidente por colisão do navio na entrada ou saída do dique, ER4 -atraso na execução dos serviços de pintura por motivos climáticos e ER5 - Não obter condições de lastro para docagem.

Mais tarde, de maneira semelhante, porém tendo como foco componentes mecânicos utilizados em docagens, foi apresentada aos especialistas uma lista contendo 121 componentes elétricos, eletrônicos e mecânicos amplamente utilizados em diversas áreas industriais extraída de [14].

O Quadro 2 elucida os sete componentes que tiveram relevância na avaliação dos especialistas e suas respectivas ocorrências. Constata-se que, também neste âmbito, os cinco primeiros componentes representam aproximadamente uma elevada percentagem de ocorrências (93,75\% de percentagem acumulada). 
Quadro 2 - Ordem dos 121 componentes mecânicos do processo de docagem

\begin{tabular}{|lccc|}
\hline \multicolumn{1}{c}{ COMPONENTES } & OCORR. & PERCENTACEM & ACUMULADO \\
\hline Motor Elétrico & 10 & $31,25 \%$ & $\mathbf{3 1 , 2 5 \%}$ \\
\hline Disjuntor do motor elétrico & 6 & $18,75 \%$ & $\mathbf{5 0 , 0 0 \%}$ \\
\hline Bomba Centrífuga para esgotamento do dique & 6 & $\mathbf{1 8 , 7 5 \%}$ & $\mathbf{6 8 , 7 5 \%}$ \\
\hline Válvula gaveta de descarga da bomba centrifuga & 5 & $15,63 \%$ & $\mathbf{8 4 , 3 8 \%}$ \\
\hline Acoplamento do motor auxiliar com a bomba de incêndio & 3 & $9,38 \%$ & $\mathbf{9 3 , 7 5 \%}$ \\
\hline Filtro de líquidos & 1 & $3,13 \%$ & $\mathbf{9 6 , 8 8 \%}$ \\
\hline Conexões elétricas & 1 & $3,13 \%$ & $\mathbf{1 0 0 , 0 0 \%}$ \\
\hline Componentes (114) não mencionados pelos especialistas & 0 & $0,00 \%$ & $\mathbf{1 0 0 , 0 0 \%}$ \\
\hline
\end{tabular}

Fonte: [8]

Como segundo critério de decisão, estes cinco primeiros componentes mecânicos foram considerados os mais significativos e consequentemente escolhidos para um estudo mais aprofundado, com os seguintes códigos: CM1 - Motor elétrico, CM2 - Disjuntor do motor elétrico, CM3 - Bomba centrífuga para esgotamento do dique, CM4 - Válvula gaveta de descarga da bomba centrífuga e CM5 - Acoplamento do motor auxiliar com a bomba de incêndio.

A seguir, para cada um dos cinco eventos de risco selecionados, foram efetuadas quatro perguntas específicas sobre os seus modos de falha, índices de severidade, ocorrência e detecção, por intermédio de um questionário com respostas em escala de Likert, com valores de 1 a 5, sendo que, quanto maior o risco, maior o valor atribuído. Vinte respostas de cada especialista foram obtidas. Este mesmo procedimento foi repetido para os cinco componentes mecânicos e vinte outras respostas de cada profissional foram fornecidas.

A partir das 1.280 escolhas, realizadas pelos 32 especialistas respondendo a 40 questões cada um, foi utilizado o método do coeficiente Alfa de Cronbach [1].

Este coeficiente varia numa escala de 0 a 1 , considerando-se satisfatórias as questões quando se atinja 0,7 como valor mínimo aceitável [1]. O coeficiente calculado de 0,71 confirma a confiabilidade do questionário.

A partir das opiniões fornecidas foi possível a elaboração de duas planilhas FMEA, sendo o Quadro 3 referente aos cinco eventos de risco e o Quadro 4 alusivo aos cinco componentes mecânicos.

Quadro 3 - Eventos de Risco 1 a 5 classificados em ordem decrescente dos respectivos RPN

\begin{tabular}{|c|c|c|c|c|c|c|c|c|}
\hline $\begin{array}{c}\text { EVENTO } \\
\text { DE RISCO }\end{array}$ & $\begin{array}{c}\text { MODO } \\
\text { DE } \\
\text { FALHA }\end{array}$ & $\begin{array}{l}\text { EFEITO (S) } \\
\text { DA FALHA }\end{array}$ & $\begin{array}{l}\text { S } \\
\text { E } \\
\text { V }\end{array}$ & $\begin{array}{l}\text { CAUSA (S) } \\
\text { DA FALHA }\end{array}$ & $\begin{array}{l}\mathbf{O} \\
\mathbf{C} \\
\mathbf{O}\end{array}$ & DETECÇÃO & $\begin{array}{l}\mathbf{D} \\
\mathbf{E} \\
\mathbf{T}\end{array}$ & $\begin{array}{l}\mathbf{R} \\
\mathbf{P} \\
\mathbf{N}\end{array}$ \\
\hline $\begin{array}{l}\text { ER1 - Falha no } \\
\text { sistema de } \\
\text { bombeamento } \\
\text { do dique }\end{array}$ & $\begin{array}{l}\text { Componente } \\
\text { emperrado } \\
\text { na posição } \\
\text { (fechada ou } \\
\text { aberta) }\end{array}$ & $\begin{array}{l}\text { Eventual parada } \\
\text { no enchimento / } \\
\text { esvaziamento do } \\
\text { dique }\end{array}$ & 4 & Detritos & 4 & Visual & 4 & 64 \\
\hline $\begin{array}{l}\text { ER2 - } \\
\text { Materiais não } \\
\text { serem } \\
\text { entregues no } \\
\text { prazo } \\
\text { solicitado }\end{array}$ & $\begin{array}{l}\text { Falha de } \\
\text { recursos } \\
\text { humanos } \\
\text { para dar } \\
\text { apoio ao } \\
\text { negócio }\end{array}$ & $\begin{array}{l}\text { Atraso no } \\
\text { recebimento de } \\
\text { materiais } \\
\text { necessários para } \\
\text { a feitura de } \\
\text { berços e } \\
\text { picadeiros }\end{array}$ & 4 & $\begin{array}{l}\text { Falha no } \\
\text { controle de } \\
\text { recursos } \\
\text { humanos }\end{array}$ & 3 & $\begin{array}{l}\text { Auditorias } \\
\text { periódicas }\end{array}$ & 5 & 60 \\
\hline
\end{tabular}




\begin{tabular}{|l|l|l|l|l|l|l|l|}
\hline $\begin{array}{l}\text { ER3 - Acidente } \\
\text { por colisão do } \\
\text { navio na } \\
\text { entrada ou } \\
\text { saída do dique }\end{array}$ & $\begin{array}{l}\text { Falha em } \\
\text { operar } \\
\text { componente }\end{array}$ & $\begin{array}{l}\text { Risco de } \\
\text { acidente }\end{array}$ & 3 & $\begin{array}{l}\text { - Falha do } \\
\text { componente } \\
\text { - Falha do } \\
\text { operador }\end{array}$ & 3 & Visual & 3 \\
\hline $\begin{array}{l}\text { ER4 - Atraso } \\
\text { na execução } \\
\text { dos serviços de } \\
\text { pintura por } \\
\text { motivos } \\
\text { climáticos }\end{array}$ & $\begin{array}{l}\text { Falta de } \\
\text { aderência da } \\
\text { tinta ao } \\
\text { casco do } \\
\text { navio }\end{array}$ & $\begin{array}{l}\text { Falha na } \\
\text { execução do } \\
\text { esquema de } \\
\text { pintura }\end{array}$ & $\begin{array}{l}\text { Temperatura do } \\
\text { casco menor que } \\
\text { a de orvalho }\end{array}$ & 3 & $\begin{array}{l}\text { Termômetro } \\
\text { laser }\end{array}$ & 3 & 18 \\
\hline $\begin{array}{l}\text { ER5 - Não } \\
\text { obter condições } \\
\text { de lastro para } \\
\text { docagem }\end{array}$ & $\begin{array}{l}\text { Falha no } \\
\text { controle de } \\
\text { pesos a } \\
\text { bordo do } \\
\text { navio }\end{array}$ & $\begin{array}{l}\text { Banda ou trim } \\
\text { no navio com } \\
\text { degradação de } \\
\text { sua estabilidade } \\
\text { estática }\end{array}$ & 3 & $\begin{array}{l}\text { Falha na } \\
\text { coordenação da } \\
\text { movimentação/ } \\
\text { adição/remoção } \\
\text { de pesos a bordo }\end{array}$ & 1 & $\begin{array}{l}\text { Auditorias } \\
\text { periódicas }\end{array}$ & 3 \\
\hline
\end{tabular}

Fonte: [8]

Quadro 4 - Componentes Mecânicos 1 a 5 classificados em ordem decrescente dos respectivos RPN

\begin{tabular}{|c|c|c|c|c|c|c|c|c|}
\hline COMPONENTE & $\begin{array}{l}\text { MODO } \\
\text { DE } \\
\text { FALHA }\end{array}$ & $\begin{array}{l}\text { EFEITO (S) } \\
\text { DA FALHA }\end{array}$ & $\begin{array}{l}\mathbf{S} \\
\mathbf{E} \\
\mathbf{V}\end{array}$ & $\begin{array}{l}\text { CAUSA (S) } \\
\text { DA FALHA }\end{array}$ & $\begin{array}{l}\mathbf{O} \\
\mathbf{C} \\
\mathbf{O}\end{array}$ & $\begin{array}{c}\text { DETECÇÃ } \\
0\end{array}$ & $\begin{array}{l}\mathbf{D} \\
\mathbf{E} \\
\mathbf{T}\end{array}$ & $\begin{array}{l}\mathbf{R} \\
\mathbf{P} \\
\mathbf{N}\end{array}$ \\
\hline $\begin{array}{l}\text { CM4 - Válvula } \\
\text { gaveta de descarga } \\
\text { da bomba centrifuga }\end{array}$ & $\begin{array}{l}\text { Haste da } \\
\text { válvula } \\
\text { danificada }\end{array}$ & $\begin{array}{l}\text { - Resposta da } \\
\text { válvula } \\
\text { deficiente } \\
\text { - Falha ao abrir } \\
\text { e/ou fechar }\end{array}$ & 4 & $\begin{array}{l}\text { - Vibração, } \\
\text { choque }\end{array}$ & 4 & Visual & 4 & 64 \\
\hline $\begin{array}{l}\text { CM1 - Motor } \\
\text { elétrico }\end{array}$ & $\begin{array}{l}\text { Enrolamen } \\
\text {-to em } \\
\text { curto }\end{array}$ & $\begin{array}{l}\text { - Motor não irá } \\
\text { partir } \\
\text { - Falha do } \\
\text { motor } \\
\text { - Faiscas nas } \\
\text { escovas }\end{array}$ & 4 & $\begin{array}{l}\text { - Repartição } \\
\text { do isolamento } \\
\text { - Alta } \\
\text { temperatura } \\
\text { ambiente } \\
\text { - Altitude } \\
\text { elevada } \\
\text { - Sobrecarga } \\
\text { mecânica } \\
\text { - Partidas e } \\
\text { paradas } \\
\text { frequentes } \\
\text { - Acúmulo de } \\
\text { sujeira em } \\
\text { aletas de } \\
\text { ventilação } \\
\text { - Vibração } \\
\text { - Choque } \\
\text { mecânico }\end{array}$ & 4 & Visual & 3 & 48 \\
\hline CM3 - Bomba & Eixo & - Deflexão e & 4 & Desgaste do & 3 & Visual & 4 & 48 \\
\hline
\end{tabular}




\begin{tabular}{|c|c|c|c|c|c|c|c|c|}
\hline $\begin{array}{l}\text { centrifuga para } \\
\text { esgotamento do } \\
\text { dique }\end{array}$ & $\begin{array}{l}\text { desbalance } \\
\text { ado }\end{array}$ & $\begin{array}{l}\text { desalinhamento } \\
\text { do eixo } \\
\text { - Vazamento } \\
\text { interno } \\
\text { Vazamento no } \\
\text { selo } \\
\text { - Desgaste do } \\
\text { mancal }\end{array}$ & & impulsor & & & & \\
\hline $\begin{array}{l}\text { CM2 - Disjuntor do } \\
\text { motor elétrico } \\
(\mathrm{CM} 1)\end{array}$ & $\begin{array}{l}\text { Contator } \\
\text { danificado }\end{array}$ & $\begin{array}{l}\text { Tensão de carga } \\
\text { excessiva }\end{array}$ & 4 & Arco contator & 3 & Visual & 3 & 36 \\
\hline $\begin{array}{l}\text { CM5 - Acoplamento } \\
\text { do motor auxiliar } \\
\text { com a bomba de } \\
\text { incêndio }\end{array}$ & $\begin{array}{l}\text { Elementos } \\
\text { flexiveis } \\
\text { ou buchas } \\
\text { do eixo } \\
\text { gastos }\end{array}$ & $\begin{array}{l}\text { Perda de } \\
\text { potencia } \\
\text { transmitida à } \\
\text { bomba / vazão } \\
\text { de saída } \\
\text { reduzida }\end{array}$ & 5 & $\begin{array}{l}\text { Excessivo } \\
\text { desalinhament } \\
\text { o do eixo }\end{array}$ & 1 & Visual & 2 & 10 \\
\hline
\end{tabular}

Fonte: [8]

Tomando por base as informações contidas nos quadros 3 e 4 foi possível confeccionar o Quadro 5 contendo a ordenação de 10 riscos a partir de seus respectivos valores de RPN. Observa-se um empate, de valores de RPN, entre ER1 e CM4 e outro entre CM1 e CM3.

Quadro 5 - Riscos classificados em ordem decrescente dos respectivos RPN

\begin{tabular}{|c|c|c|}
\hline $\begin{array}{c}\text { Evento de Risco/ } \\
\text { Componente } \\
\text { Mecânico }\end{array}$ & RPN & $\begin{array}{c}\text { Classificação } \\
\text { Hierárquica }\end{array}$ \\
\hline ER1 & 64 & 1 \\
\hline CM4 & 64 & 1 \\
\hline ER2 & 60 & 2 \\
\hline CM1 & 48 & 3 \\
\hline CM3 & 48 & 3 \\
\hline CM2 & 36 & 4 \\
\hline ER3 & 27 & 5 \\
\hline ER4 & 18 & 6 \\
\hline CM5 & 10 & 7 \\
\hline ER5 & 9 & 8 \\
\hline
\end{tabular}

Fonte: [8]

Na intenção de realizarmos uma classificação dos 10 riscos identificados no Quadro 5 com uma melhor ordenação entre eles, foi aplicado o método de composição probabilística de preferências (CPP). A CPP se baseia na substituição das avaliações numéricas iniciais em probabilidades de cada opção ser a mais importante segundo cada critério. Esta transformação probabilística é particularmente adequada à FMEA porque acentua as distâncias entre as avaliações de risco mais alto e reduz a distância entre as avaliações de risco inferior. 
Com este método, baseando-se nas avaliações numéricas de severidade, ocorrência e detecção realizadas pelos especialistas, se efetua a priorização das falhas potenciais através do cálculo do número de prioridade probabilística (NPP), definido como o produto das probabilidades de preferência segundo cada critério. As avaliações pelos especialistas permitiram associar a cada avaliação segundo um diferente fator de risco uma distribuição triangular com a moda estimada pela moda observada no conjunto das 32 avaliações.

A referência [17] menciona que o limite do valor de risco é determinado através do cálculo de $1 / n^{3}$, onde $n$ representa o número de modos de falha contidos nas planilhas de FMEA.

No caso, como os 5 eventos de risco (ER) e os 5 componentes mecânicos (CM) possuem 5 modos de falha cada um, e, seguindo (NSWC 2011), consideramos para o CM2 (Disjuntor do motor elétrico), no modo de falha "Bobina queimada", duas distintas causas e efeitos, lidamos com $n=51$. Por isto, utiliza-se $n=51$ no Quadro 6.

Assim, riscos cujos valores de NPP sejam superiores a este limite $(51-3 \approx 7,5386 \mathrm{E}$ 06) deverão ser tratados. O Quadro 6 abaixo elucida os padrões probabilísticos estipulados.

Quadro 6 - Padrões probabilísticos de riscos

\begin{tabular}{c|c|c|c|c}
\hline$\leq n^{-3} / 3$ & $>n^{-3} / 3 e \leq 2 n^{-3} / 3$ & $>2 n^{-3} / 3 e \leq n^{-3}$ & $>n^{-3} e \leq 3 n^{-3} / 2$ & $>3 n^{-3} / 2$ \\
\hline Muito baixo & Baixo & Médio & Alto & \multirow{2}{*}{ Muito alto } \\
\hline
\end{tabular}

Fonte: [17]

A partir do cálculo da composição probabilística referente aos cinco eventos de risco do Quadro 3 e aos cinco componentes mecânicos do Quadro 4, obteve-se o Quadro 7, que representa a listagem dos riscos dos modos de falha selecionados, em ordem decrescente dos valores de seus respectivos NPP, as respectivas prioridades para tratamento e o ponto de corte assinalado.

Quadro 7 - Ordenamento de 10 riscos utilizando a Composição probabilística de preferência

\begin{tabular}{|c|c|c|c|}
\hline $\begin{array}{c}\text { Evento de Risco/ } \\
\text { Componente } \\
\text { Mecânico }\end{array}$ & NPP & $\begin{array}{c}\text { Prioridade para } \\
\text { Tratamento }\end{array}$ & $\begin{array}{c}\text { Padrão } \\
\text { Probabilístico }\end{array}$ \\
\hline ER1 & $2,27534 \mathrm{E}-04$ & 1,5 & Muito Alto \\
\hline CM4 & $2,27534 \mathrm{E}-04$ & 1,5 & Muito Alto \\
\hline ER2 & $1,76186 \mathrm{E}-04$ & 3 & Muito Alto \\
\hline CM1 & $1,30162 \mathrm{E}-04$ & 4 & Muito Alto \\
\hline CM3 & $5,99504 \mathrm{E}-05$ & 5 & Muito Alto \\
\hline CM2 & $3,42948 E-05$ & 6 & Muito Alto \\
\hline CM5 & $2,75345 E-05$ & 7 & Muito Alto \\
\hline ER3 & $1,96083 E-05$ & 8 & Muito Alto \\
\hline ER4 & $1,37338 E-05$ & 9 & Muito Alto \\
\hline ER5 & $7,57154 E-06$ & 10 & Alto \\
\hline \multicolumn{2}{|c|}{ Ponto de Corte: $\mathrm{n}-3 \cong 7,5386 \mathrm{E}-06$} & \\
\hline
\end{tabular}

Fonte: [8] 
Observa-se no Quadro 7 que os nove primeiros riscos possuem um padrão probabilístico considerado "muito alto" (NPP > 3*51-3/2 $\approx 1,13079 \mathrm{E}-05$ ) e o último, ER5, um padrão "alto". A inclusão de outros critérios de análise poderá promover o desempate entre as prioridades atribuídas aos ER1 e CM4.

Realizando-se um cotejamento entre os quadros 5 e 7, observa-se que a vantajosa e robusta aplicação do método da composição probabilística, proposto por [17], gerou distintos valores de NPP para oito riscos, os quais propiciaram uma melhor priorização para os seus tratamentos, quando comparado ao calculo do RPN da FMEA clássica, em que ocorreram dois empates de RPN. O risco CM5 subiu da nona posição (quadro 5) para a sétima (quadro 7) na sua prioridade para tratamento, porém permaneceu como ultima prioridade caso consideremos somente os componentes mecânicos.

Como resultado do estudo empreendido, os fatores críticos identificados são cinco eventos de risco e cinco componentes mecânicos descritos, respectivamente, nos quadros 8 e 9 e hierarquizados em ordem decrescente dos seus valores de NPP transcritos do Quadro 7.

Quadro 8 - Eventos de risco críticos para a análise do modo de falha e efeitos

\begin{tabular}{|c|l|c|}
\hline CÓDIGO & \multicolumn{1}{|c|}{ EVENTOS DE RISCO } & NPP \\
\hline ER1 & Falha no sistema de bombeamento do dique & $2,27534 \mathrm{E}-04$ \\
\hline ER2 & Materiais não serem entregues no prazo solicitado & $1,76186 \mathrm{E}-04$ \\
\hline ER3 & Acidente por colisão do navio na entrada ou saída do dique & $1,96083 \mathrm{E}-05$ \\
\hline ER4 & Atraso na execução dos serviços de pintura por motivos climáticos & $1,37338 \mathrm{E}-05$ \\
\hline ER5 & Não obter condições de lastro para docagem & $7,57154 \mathrm{E}-06$ \\
\hline
\end{tabular}

Fonte: [8]

Quadro 9 - Componentes mecânicos críticos para a análise do modo de falha e efeitos

\begin{tabular}{|c|l|c|}
\hline CÓDIGO & \multicolumn{1}{|c|}{ COMPONENTES MECÂNICOS } & NPP \\
\hline CM4 & Válvula gaveta de descarga da bomba centrífuga & $2,27534 \mathrm{E}-04$ \\
\hline CM1 & Motor elétrico & $1,30162 \mathrm{E}-04$ \\
\hline CM3 & Bomba centrífuga para esgotamento do dique & $5,99504 \mathrm{E}-05$ \\
\hline CM2 & Disjuntor do motor elétrico & $3,42948 \mathrm{E}-05$ \\
\hline CM5 & Acoplamento do motor auxiliar com a bomba de incêndio & $2,75345 \mathrm{E}-05$ \\
\hline
\end{tabular}

Fonte: [8]

A partir de uma revisita realizada em abril/2019 ao mesmo dique seco estudado, cerca de quatro anos atrás por [8], foram levantados, por meio de entrevistas realizadas aos 
especialistas responsáveis pela área de docagem, os eventos de risco atualmente existentes em operações de docagem de navios, descritos no quadro 10.

Quadro 10 - Eventos de risco críticos resultantes de revisita realizada em abril/2019

\begin{tabular}{|c|l|}
\hline CÓDIGO & \multicolumn{1}{|c|}{ EVENTOS DE RISCO APÓS REVISITA } \\
\hline ER1 & Risco de “Falha no sistema de bombeamento do dique” foi tratado \\
\hline ER2 & Risco de “Materiais não serem entregues no prazo solicitado” foi tratado \\
\hline ER3 & Acidente por colisão do navio na entrada ou saída do dique \\
\hline ER4 & Atraso na execução dos serviços de pintura por motivos climáticos \\
\hline ER5 & Risco de “Não obter condições de lastro para docagem” foi tratado \\
\hline ER6 & $\begin{array}{l}\text { Impossibilidade de entrada ou saída por condições ambientais desfavoráveis } \\
\text { (maré, vento) }\end{array}$ \\
\hline ER7 & Indisponibilidade de rebocadores para a docagem \\
\hline ER8 & Falta de energia elétrica \\
\hline
\end{tabular}

Fonte: Os autores

De modo semelhante, foram levantados os componentes mecânicos críticos atualmente existentes em operações de docagem de navios, descritos no Quadro 11.

Quadro 11 - Componentes mecânicos críticos resultantes de revisita realizada em abril/2019

\begin{tabular}{|c|l|}
\hline CÓDIGO & \multicolumn{1}{|c|}{ COMPONENTES MECÂNICOS APÓS REVISITA } \\
\hline CM4 & Válvula gaveta de descarga da bomba centrífuga \\
\hline CM1 & Motor elétrico \\
\hline CM3 & Risco de "Bomba centrífuga para esgotamento do dique” foi tratado \\
\hline CM2 & Risco do “Disjuntor do motor elétrico" foi tratado \\
\hline CM5 & $\begin{array}{l}\text { Risco do “Acoplamento do motor auxiliar com a bomba de incêndio” foi } \\
\text { tratado }\end{array}$ \\
\hline CM6 & Mancal (temperatura elevada) \\
\hline CM7 & Cabos de alimentação (ação de ratos) \\
\hline
\end{tabular}

Fonte: Os autores 
A partir da comparação entre os quadros 8 e 10, observa-se que os eventos de risco (ER) 1, 2 e 5 foram tratados pelo estaleiro, os ER 3 e 4 permaneceram inalterados por serem afetos a condições da própria natureza e os ER 6, 7 e 8 foram acrescentados.

Com base no cotejamento entre os quadros 9 e 11, constata-se que os componentes mecânicos (CM) críticos 1 e 2 permaneceram inalterados, os CM 3, 4 e 5 foram tratados pelo estaleiro e os CM 6 e 7 foram acrescentados.

\section{CONSIDERAÇÕES FINAIS}

O estudo de [8] foi precursor na análise dos riscos existentes em operações de docagem de navios em diques secos através da aplicação conjunta da técnica de Análise de Riscos Qualitativa, da FMEA e da CPP.

Para a sua consecução foram desenvolvidas as seguintes atividades: revisão bibliográfica, escolha da técnica de análise de riscos a ser utilizada, definição de uma metodologia de pesquisa, abordagem bibliométrica, identificação dos eventos de risco e componentes mecânicos inerentes à docagem, aplicação de um survey junto a 32 especialistas, identificação dos modos de falha, índices de severidade, ocorrência e detecção, validação do survey, preenchimento de dez planilhas de FMEA e identificação de riscos prioritários.

Como resultado decorrente da pesquisa realizada, observou-se que a aplicação do método da composição probabilística gerou valores que propiciaram uma melhor priorização para tratamento dos riscos considerados inaceitáveis, quando comparado ao calculo do RPN da FMEA clássica, e uma robustez ao processo de tomada de decisões nos cenários de docagem de navios.

A proposta deste artigo é revisitar, decorridos cerca de quatro anos, por meio de entrevistas realizadas perante os especialistas responsáveis pela área de docagem, os riscos atualmente existentes em operações de docagem de navios, especificamente no mesmo dique seco estudado em 2015, em que, contextos e dados então vigentes agora, se verificaram desatualizados.

Embora efeitos financeiros não façam parte do escopo deste texto, estes deverão ser abordados em outro estudo por ocasião da tomada de decisões deste tipo.

A partir do desenvolvimento deste artigo, através de uma revisita ao mesmo dique seco estudado por [8], espera-se um fomento no gerenciamento de riscos nas operações de docagem de navios em diques secos, contribuindo sobremaneira para o fortalecimento das forças de superfície e de submarinos da Marinha, frota mercante e consequentemente da soberania brasileira na Amazônia azul.

\section{REFERÊNCIAS BIBLIOGRÁFICAS}

[1] ALMEIDA, D.; SANTOS, M.; COSTA, A. Aplicação do Coeficiente Alfa de Cronbach nos resultados de um questionário para avaliação de desempenho da saúde pública. In: XXX ENCONTRO NACIONAL DE ENGENHARIA DE PRODUÇÃO ENEGEP 2010, São Carlos, SP, 2010.

[2] ANTAQ. AGÊNCIA NACIONAL DE TRANSPORTES AQUAVIÁRIOS. Disponível em: http://web.antaq.gov.br/Portal/Portos_Apresentacao.asp. Acesso em: 18 ABR 2019.

[3] CICEK, K., CELIK, M. Application of Failure Modes and Effects Analysis to Main Engine Crankcase Explosion Failure on-Board Ship. Safety Science, v.51, n.1, p. 610, 2013. 
[4] FERREIRA, A. B. H. Novo Dicionário Aurélio da Língua Portuguesa. 3. ed., Curitiba, Editora Positivo, 2012.

[5] GEIGER, P. Novíssimo Aulete - Dicionário Contemporâneo da Língua Portuguesa. Editora Lexikon, 2013.

[6] GIL, A.C. Como Elaborar Projetos de Pesquisa. 5a. Ed. São Paulo: Atlas, 1999.

[7] HOLLNAGEL, E. The Changing Nature of Risk. ERGONOMICS AUSTRALIA JOURNAL, 22 (1-2), PP.33-46, 2008.

[8] KÄMPFFE, M. G. F., et al. Apoio à Decisão Baseada em Risco: Aplicação de Instrumento Híbrido na Docagem de Embarcações em Diques Secos. REVISTA PESQUISA NAVAL NUMERO 27, 2015.

[9] KÄMPFFE, M. G. F. Análise de Riscos aplicada à Docagem de Navios em Diques Secos: um Estudo da Aplicação da Técnica de Análise de Modos de Falha e Efeitos, Disponível em: https://sucupira.capes.gov.br/sucupira/public/consultas/coleta/trabalho Conclusao/viewTrabalhoConclusao.jsf?popup=true\&id_trabalho=1361715.

Dissertação de Mestrado, Universidade Federal Fluminense, 2014.

[10] LEES, F. P. Loss Prevention in The Process Industries: Hazard Identification, Assessment and Control. Oxford, Butterworth-Heinemann, 2.ed., 1996.

[11] LIMA, G. Uma Abordagem Multicritério para a Avaliação do Grau de Risco dos Ramos de Atividade Econômica. Tese de Doutorado em Engenharia apresentada ao curso de Pós-Graduação de Engenharia da Universidade Federal do Rio de Janeiro, COPPE/UFRJ, Rio de Janeiro, 2001.

[12] LIU, H. C.; LIU, L.; LIU, N. Risk Evaluation Approaches in Failure Mode and Effects Analysis: A Literature Review. In: EXPERT SYSTEMS WITH APPLICATIONS, n.32; p.828-838, 2013.

[13] MODICA, J. E. - Riscos em Projetos de Docagens de Navios Petroleiros. 2009. 121p. Dissertação (Mestrado) - Escola Politécnica, Universidade de São Paulo. USP, São Paulo, 2009.

[14] NAVAL SURFACE WARFARE CENTER (NSWC) - Handbook of Reliability Prediction Procedures for Mechanical Equipment, United States, Department of the Navy, Washington D.C., 2011.

[15] PMI - PROJECT MANAGEMENT INSTITUTE. Standards Committee. Conjunto de Conhecimentos em Gerenciamento de Projetos (PMBOK). 3.ed. Newton Square, 2004.

[16] SANT’ANNA, A. P; PINTO JR, R. Composição Probabilística no Cálculo das Prioridades na FMEA. In: REVISTA ELETRÔNICA SISTEMAS \& GESTÃO 5 (3) 179-191, 2010.

[17] SANT'ANNA, A. P. Probabilistic Priority Numbers for Failure Modes and Effects Analysis. In: INTERNATIONAL JOURNAL OF QUALITY \& RELIABILITY MANAGEMENT. Vol 29 no. 3, p. 349-362. 2012.

[18] SANT’ANNA, A. P. Procedimento de Cálculo para a Composição Probabilística de Preferências. In: RELATÓRIOS DE PESQUISA EM ENGENHARIA DE PRODUÇÃO, v.13, Série C, n. 1, p. 1-11, 2013. 
[19] SANT'ANNA, A. P. Probabilistic Composition of Preferences, Theory and Applications. Springer International Publishing Switzerland., 2015.

[20] SANT’ANNA, A. P.; LIMA, G.; GAVIAO, L. O. A Probabilistic Approach to the Inequality Adjustment of the Human Development Index. In: PESQUISA OPERACIONAL 38(1): 99-116, 2018.

[21] SILVA, A. M. B. C. Leis de Pareto Para Guerras e Actos de Terrorismo. Dissertação em Engenharia Eletrotécnica e de Computadores. Departamento de Engenharia Electrotécnica do Instituto Superior de Engenharia do Porto, 2014.

[22] SOUZA, J. Gestão de Projeto para Docagem de Navios em Diques. Monografia Universidade Candido Mendes. UCAM, RJ, 2010.

[23] U. S. DEPARTMENT OF DEFENSE - MIL-STD-1629A - Procedures for Performing a Failure Mode, Effects and Criticality Analysis, Military Standard, Naval Ship Engineering Center, Washington D.C., 1980. 